\title{
HYPERPROLACTINEMIA AND LEYDIG CELL TUMOR
}

16-20 May 2015

DUBLIN, IRELAND

TTh EUROPEAN CONGRESS OF ENDOCRINOLOGY
Ana Margarida Monteiro, Marta Alves, Olinda Marques

Endocrinology Service of Hospital de Braga

\section{INTRODUCTION}

Leydig cell tumors are the most common testicular sex cord stromal tumors. The most frequent clinical presentation is a testicular mass. However, they can present with endocrine manifestations, and gynecomastia is the most common presentation. Hyperprolactinemia can cause hypogonadotropic hypogonadism, and in males, is also a cause of gynecomastia.

\section{CLINICAL CASE}

A 24-years-old male was referred to our Endocrinology department due to hyperprolactinemia and increasing bilateral breast volume with a year of evolution, without galactorrhoea, libido changes or erectile dysfunction.

He had irrelevant medical past history, without medication, smoking, alcohol or illicit drug use.

On physical examination, bilateral painful gynecomastia, without palpable nodules, skin changes or nipple retraction or galactorrhea. Testes were present, with no palpable nodules.

Laboratory exams revealed normal testosterone and FSH, slightly low LH, high estradiol (although normal in its first evaluation) and high prolactin levels. TSH was normal and testicular tumour markers were normal. (table 1 )

Breast ultrasound showed increased bilateral mammary glands, compatible with gynecomastia.

The initial pituitary MRI showed an area of low uptake which could correspond to a microadenoma.

He started bromocriptine $2.5 \mathrm{mg} /$ day.

In the scrotal ultrasound an isoechogenic nodule with $12 \mathrm{~mm}$ in the right testicle was observed.

Referenced to urology consultation

Scrotal MRI confirmed the nodule in the right testicle (image 1).

He was submitted to right partial orchiectomy guided by ultrasound, and histological examination revealed a Leydig cell tumor.

After surgery, we stopped bromocriptine use and pituitary MRI was normal (image 2). Currently, he has normal prolactin levels (table 2).

He was oriented to plastic surgery to bilateral mastectomy.

\begin{tabular}{|c|c|c|c|c|c|}
\hline \multicolumn{2}{|l|}{ Table 1} & \multicolumn{2}{|c|}{ Results } & \multicolumn{2}{|l|}{$\mathbf{N}$} \\
\hline \multicolumn{2}{|l|}{$\mathrm{FSH}(\mathrm{mUl} / \mathrm{mL})$} & \multicolumn{2}{|l|}{ 1,44 } & \multicolumn{2}{|l|}{$1.4-18.1$} \\
\hline \multicolumn{2}{|l|}{$\mathrm{LH}(\mathrm{mUl} / \mathrm{mL})$} & \multicolumn{2}{|c|}{1,43} & \multicolumn{2}{|l|}{$1.5-9.3$} \\
\hline \multicolumn{2}{|c|}{ Testosterone (ng/dL) } & \multicolumn{2}{|l|}{263,46} & \multicolumn{2}{|l|}{$241-827$} \\
\hline \multicolumn{2}{|l|}{ Estradiol (pmol/L) } & \multicolumn{2}{|c|}{$206,02 \uparrow$} & \multicolumn{2}{|l|}{$<146.1$} \\
\hline \multicolumn{2}{|l|}{ TSH (uUI/mL) } & \multicolumn{2}{|c|}{1,90} & \multicolumn{2}{|l|}{$0.358-3.74$} \\
\hline \multicolumn{2}{|l|}{ FT4 (ng/dL) } & \multicolumn{2}{|c|}{1,12} & \multicolumn{2}{|l|}{$0.76-1.46$} \\
\hline \multicolumn{2}{|c|}{ Prolactin $0^{\prime} / 15^{\prime} / 30^{\prime}(\mathrm{ng} / \mathrm{mL})$} & \multicolumn{2}{|c|}{$27,08 / 35,49 / 30,30 \uparrow$} & \multicolumn{2}{|l|}{$2.1-17.7$} \\
\hline \multicolumn{2}{|c|}{ Creatinine (mg/dl) } & \multicolumn{2}{|c|}{0,6} & \multicolumn{2}{|l|}{$0,70-1,20$} \\
\hline \multicolumn{2}{|l|}{ AST (U/L)0 } & \multicolumn{2}{|c|}{14} & \multicolumn{2}{|l|}{$15-37$} \\
\hline \multicolumn{2}{|l|}{ ALT (U/L) } & \multicolumn{2}{|c|}{28} & \multicolumn{2}{|l|}{$12-78$} \\
\hline \multicolumn{2}{|l|}{ GGT (U/L) } & \multicolumn{2}{|c|}{12} & \multicolumn{2}{|l|}{$15-85$} \\
\hline Alkaline phosphata & se (u/L) & 56 & & 50-136 & \\
\hline Bilirubin (mg/dl) & & 0,43 & & $0,1-1,0$ & \\
\hline B-hCG & & $<0,1$ & & & \\
\hline Table 2 & $\begin{array}{l}\text { Results } \\
-4 \text { months after } \\
\text { bromocriptine suspe } \\
-16 \text { months after su }\end{array}$ & $\begin{array}{l}\text { pension } \\
\text { urgery }\end{array}$ & $\begin{array}{l}\text { Results } \\
-4 \text { months after surgery } \\
- \text { on bromocriptine }\end{array}$ & $\begin{array}{l}\text { Results } \\
\text { - on } \\
\text { bromocriptine }\end{array}$ & $\mathrm{N}$ \\
\hline $\mathrm{LH}(\mathrm{mUl} / \mathrm{mL})$ & 3,36 & & 7,09 & 1,58 & $1.5-9.3$ \\
\hline Testosterone (ng/dL) & 547,46 & & 596,93 & 296,06 & $241-827$ \\
\hline Estradiol (pmol/L) & 109,51 & & 120,90 & $202,35 \uparrow$ & $<146.1$ \\
\hline Prolactin $(\mathrm{ng} / \mathrm{mL})$ & 14,75 & & 7,43 & 7,91 & 2.1-17.7 \\
\hline
\end{tabular}

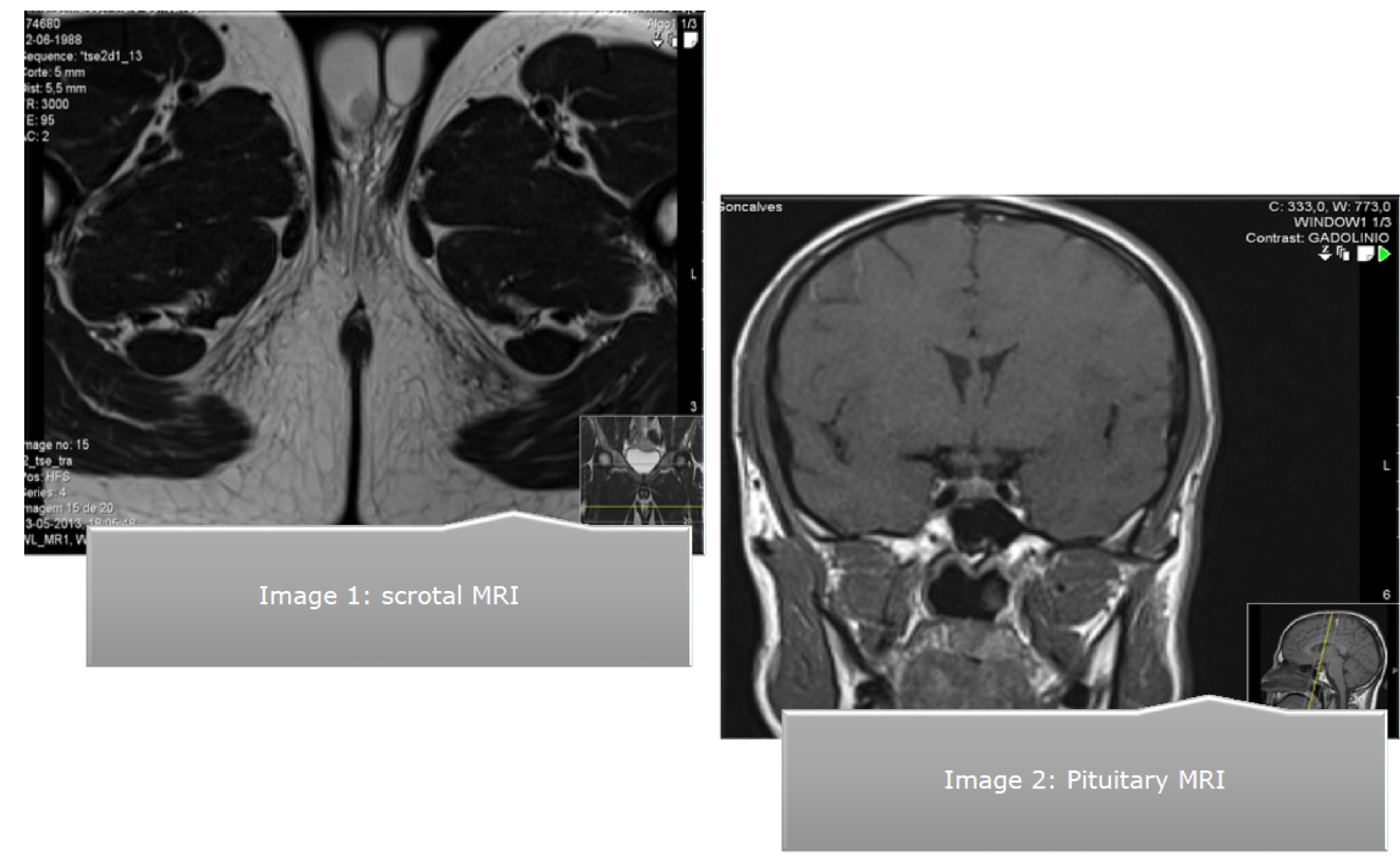

\section{DISCUSSION}

Prolactinomas and Leydig cells tumors are possible causes of gynecomastia and hyperprolactinemia (in the last because of hyperestrogenism) making it sometimes a challenge the evaluation of prolactin levels in this context.

\section{REFERENCES}

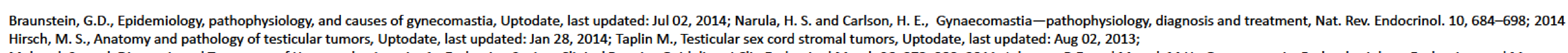

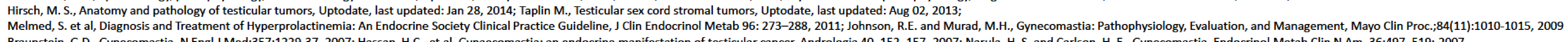

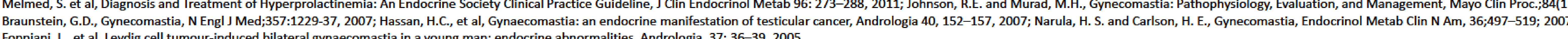

